\title{
Efficacy and safety of continuous infusion of Rh-endostatin combined with platinum-based chemotherapy for advanced triple-negative breast cancer
}

\author{
Aihua Tan, Hongxue Wang, Li Nong, Yuxian Jia, Yan Liu, Wuning Zhong, Fanghui Qin, Han Wang, \\ Jing Tang, Wenxian Zhou, Yongkui Lu, Weimin Xie \\ Department of Breast, Bone \& Soft Tissue Oncology, Guangxi Medical University Cancer Hospital, Nanning, China \\ Contributions: (I) Conception and design: W Xie, Y Lu, A Tan; (II) Administrative support: W Xie, Y Lu; (III) Provision of study materials or patients: \\ A Tan, H Wang, L Nong, Y Jia, W Zhong, F Qin, H Wang, J Tang; (IV) Collection and assembly of data: A Tan, Y Liu; (V) Data analysis and \\ interpretation: W Xie, Y Lu, A Tan; (VI) Manuscript writing: All authors; (VII) Final approval of manuscript: All authors. \\ Correspondence to: Weimin Xie. Department of Breast, Bone \& Soft Tissue Oncology, Guangxi Medical University Cancer Hospital, Nanning 530021, \\ China. Email: xieweimin1358@outlook.com.
}

\begin{abstract}
Background: This study aimed to prospectively evaluate and investigate the efficacy and safety of recombinant human endostatin (Rh-endostatin) combined with platinum-based regimens for advanced triple-negative breast cancer (TNBC) patients.
\end{abstract}

Methods: This study was a prospective, single-arm, single-center, open-label trial. From January 2017 to August 2019, 21 women aged 18-70 years with histologically confirmed advanced TNBC were enrolled. Rhendostatin at $30 \mathrm{mg} / \mathrm{d}$ was continuously pumped for 7 days and used synchronously with the chemotherapy cycle. The primary endpoint of this study was progression-free survival (PFS). Secondary endpoints included objective response rate (ORR), disease control rate (DCR), clinical benefit rate (CBR), overall survival (OS), and toxicity.

Results: The median PFS (mPFS) was 8.8 months (95\% CI: 7.2-10.4 months), and the median OS was 13.3 months (95\% CI: 11.6-15.0 months). The ORR and CBR for the whole population were $47.6 \%$ and $52.4 \%$, respectively. Patients sensitive to anthracycline and taxane drugs showed a significantly longer mPFS compared to those who were resistant to anthracycline and taxane drugs (mPFS: 8.8 vs. 5.3 months, $\mathrm{P}=0.038$ ). For patients who received first- and second-line therapy or beyond, the mPFS was 8.8 and 5.3 months, respectively, with a significant difference $(\mathrm{P}=0.025)$. No statistically significant differences in the $\mathrm{mPFS}$ between pemetrexed combined with platinum and gemcitabine/taxanes combined with platinum were observed. The most common grade 3-4 hematologic toxicities were neutropenia (14.3\%) and anemia (14.3\%). One patient (4.8\%) experienced febrile neutropenia. No grade 3-4 non-hematologic toxicities were observed, and no treatment-related deaths were reported in this study.

Conclusions: This study revealed that Rh-endostatin might enhance the antitumor effects of platinumbased chemotherapy for advanced TNBC patients with well-tolerated toxicities, which may provide a new basis and novel idea for the treatment of TNBC. However, further investigations and validation of its longterm efficacy and toxicity are warranted in the future.

Keywords: Triple-negative breast cancer (TNBC); recombinant human endostatin (Rh-endostatin); platinumbased chemotherapy; efficacy

Submitted Aug 30, 2021. Accepted for publication Nov 25, 2021.

doi: 10.21037/apm-21-2624

View this article at: https://dx.doi.org/10.21037/apm-21-2624 


\section{Introduction}

Triple-negative breast cancer (TNBC) is a type of breast cancer (BC) in which immunohistochemical markers such as estrogen receptor (ER), progesterone receptor $(\mathrm{PgR})$, and human epidermal growth factor receptor 2 (HER2) are all negative, accounting for about $15-20 \%$ of all BCs. Compared with other types of $\mathrm{BC}, \mathrm{TNBC}$ is characterized by poor prognosis, strong invasiveness, and early recurrence and metastasis (1).

Due to the lack of effective targets for endocrine therapy and anti-HER2 therapy, systemic chemotherapy is the main treatment for patients with advanced TNBC (2). For $\mathrm{BC}$, anthracyclines and taxanes have remained the main options for chemotherapy, while they were often used in neoadjuvant or adjuvant chemotherapy. There is no standard treatment for TNBC with late recurrence and metastasis. Previous evidence reported that more than $75 \%$ of BC susceptibility gene (BRCA)1/2-mutated BCs showed the TNBC phenotype (3). Therefore, TNBC may show greater sensitivity to platinum drugs, and platinum-based chemotherapy might be an effective therapy for advanced TNBC.

Angiogenesis, which plays a crucial role in the processes of tumor invasion, growth, and metastasis, has been considered as a potentially important target for cancer treatment (4). Compared with the other types of BC, the expression levels of vascular endothelial growth factor (VEGF) and microvascular density in TNBC were significantly increased, which supports the anti-angiogenesis therapy of advanced TNBC $(5,6)$. Recombinant human endostatin (Rh-endostatin/Endostar ${ }^{\circledR}$ ), a new biological product of angiostatin, is a multiple inhibitor of angiogenesis which shows its anti-angiogenic activity by blocking a $5 \mathrm{p} 1$ integrin (cell adhesion molecule in angiogenesis), endothelin, and the activity of metalloproteinases 2, 9 and 13 (7). So far, extensive clinical trials have confirmed the significant efficacy and survival benefits of Rh-endostatin for treatment of advanced non-small cell lung cancer (NSCLC), which was approved by National Medical Products Administration (NMPA) for treatment of NSCLC patients in September of 2005. Besides, increasing bodies of clinical studies and experiences explored and uncovered the application of Rh-endostatin to treat a variety of different cancers, including colon cancer, melanoma, gastric cancer, BC, NSCLC in other setting, including serous effusion and others with a certain therapeutic effect (8). And clinical data of Rh-endostatin including its survival benefits, optimized dosages, routes of administration, recommended duration and frequency of treatment, predictive biomarkers, and its safety profile in lung cancers as well as other cancers were also explored.

In BC, development and achievement of agents that inhibit tumor angiogenesis has been an active area of investigation. Increasing evidences revealed that patients with TNBC may have a greater probability of obtaining some kind of clinical efficacy benefit from anti-angiogenicbased therapies. To date, a few clinical studies investigated the roles of Rh-endostatin in BC treatment preliminarily. Huang et al. reported that Rh-endostatin plus taxanebased regimens were effective and safe for HER2 negative metastatic BC (9). Chen et al. and Zhang et al. reported that Rh-endostatin added to neoadjuvant chemotherapy showed a better clinical efficacy in treatment of locally advanced BC, respectively $(10,11)$. However, there were few studies on the treatment of advanced TNBC with Rhendostatin plus chemotherapy. Considering these promising data of the potential therapeutic benefits of combination of Rh-endostatin and platinum-based chemotherapy, this prospective study was conducted to investigate the efficacy and safety of Rh-endostatin combined with platinum-based chemotherapy for advanced TNBC, aiming to explore and uncover alternative treatment strategy for TNBC patients.

We present the following article in accordance with the TREND reporting checklist (available at https://dx.doi. org/10.21037/apm-21-2624).

\section{Methods}

\section{Patients}

The included patients had inoperable local recurrence or metastatic BC, with a negative ER, PgR, and HER2 primary breast tumor lesion or new metastases. HER2 negative was defined as no staining or scores of $1+$ by immunohistochemistry (IHC), and cases with scores of $2+$ by IHC were confirmed by the absence of HER2 gene amplification with fluorescence in-situ hybridization (FISH). Hormone receptor negative was defined as $<1 \%$ positive tumor cells by immunohistochemical nuclear staining of both ER and PgR. All of the patients had a life expectancy of more than 3 months, and were required to have at least one measurable disease according to the Response Evaluation Criteria in Solid Tumors (RECIST version 1.1) criteria. All patients had the Eastern Cooperative Oncology Group (ECOG) performance status $\leq 2$, with adequate hematologic, 
renal, and hepatic function, which were indicated as hemoglobin $\geq 8 \mathrm{~g} / \mathrm{dL}$, absolute neutrophil count (ANC) $\geq 1.5 \times 10^{9} / \mathrm{L}$, platelet count $\geq 100 \times 10^{9} / \mathrm{L}, \mathrm{AST} / \mathrm{ALT} \leq 2.5 \times$ upper limit of normal (ULN) $(\leq 5 \times$ ULN in case of liver metastases), total serum bilirubin $\leq 1.5 \times \mathrm{ULN}$, and serum creatinine $\leq 1.0 \times$ ULN. Patients were excluded if they had symptomatic brain metastases, uncontrolled hypertension (systolic blood pressure higher than $180 \mathrm{mmHg}$ or diastolic blood pressure higher than $100 \mathrm{mmHg}$ ), or serious infections. After review and approval by the local Ethics Committee, a total of 21 patients were finally enrolled in this study. All of the enrolled patients signed the informed consent and could actively cooperate with the treatment. The study was approved by the Medical Ethics Committee of Guangxi Medical University Cancer Hospital (No. LW2021099) and was conducted in accordance with the Declaration of Helsinki (as revised in 2013). The study protocol has been registered in the Chinese Clinical Trial Registry (Identifier: ChiCTR-OIC-16009690) (https://cdn. amegroups.cn/static/public/APM-21-2624-1.pdf).

\section{Intervention measures}

Platinum drugs included carboplatin area under the curve (AUC) 5 or cisplatin $75 \mathrm{mg} / \mathrm{m}^{2}$. The combined chemotherapy included gemcitabine $1 \mathrm{~g} / \mathrm{m}^{2} \mathrm{~d} 1,8$, docetaxel $75 \mathrm{mg} / \mathrm{m}^{2}$, or pemetrexed $500 \mathrm{mg} / \mathrm{m}^{2}$. The chemotherapy was performed once every 3 weeks and continued until progressive disease (PD), unacceptable toxicity, consent withdrawal, or up to 6-8 cycles, whichever came first. The use of chemotherapy drugs referred to the drug manual. Dose modifications of chemotherapy drugs were made according to the clinical routine. Administration of prophylactic granulocyte colony stimulating factor (G-CSF) was permitted. Rh-endostatin at $30 \mathrm{mg} / \mathrm{d}$ was continuously pumped for 7 days (12) and used synchronously with the chemotherapy cycle until PD, unacceptable toxicity, or consent withdrawal, whichever came first. No reduction in $\mathrm{Rh}$-endostatin dose was permitted.

\section{Baseline and follow-up assessment}

The assessment of patients before treatment included a detailed history, physical examination, laboratory tests and the performance status. Laboratory tests included routine blood tests, liver function, renal function, electrolytes, and urinalysis. Computed tomography (CT) scans or magnetic resonance imaging (MRI) was conducted for efficacy evaluation at baseline, every 2 treatment cycles up to $6-8$ cycles, and every $2-3$ months thereafter until disease progression or death.

\section{Clinical efficacy}

The primary endpoint of this study was progressionfree survival (PFS), and the secondary endpoints were the objective response rate (ORR), clinical benefit rate (CBR), overall survival (OS), and safety. PFS was defined as the time from patient enrollment to the first recorded disease progression or death (whichever occurred first). OS was defined as the time from patient enrollment to death (from any cause of death). Clinical efficacy was divided into complete response (CR), partial response (PR), stable disease (SD), and PD, and evaluated according to the RECIST version 1.1 criteria (13). The ORR was calculated as $\mathrm{CR}+\mathrm{PR}$, the disease control rate (DCR) was calculated as $\mathrm{CR}+\mathrm{PR}+\mathrm{SD}$, and the CBR was calculated as $(\mathrm{CR}+$ $\mathrm{PR}+\mathrm{SD}$ ) $>6$ months. Adverse events (AEs) were recorded at each cycle until 30 days after the last dose of a study treatment was administered. Toxicity was evaluated and graded in line with the National Cancer Institute Common Terminology Criteria for AEs (version 4.0) (14).

\section{Statistical analysis}

All data were analyzed with the SPSS version 18 (SPSS Inc., Chicago, IL, USA). The categorical data was described as frequency or percentage (\%), and the chi-square test or Fisher's exact probability method was used for comparisons between groups. The Kaplan-Meier method and log-rank test were used for survival analysis. The Cox proportional hazard regression model was used for prognosis analysis, and stratified analysis was performed according to the number of treatment lines, the combined treatment regimens, and sensitivity to anthracycline or taxane treatment. $\mathrm{P}<0.05$ was considered statistically significant.

\section{Results}

\section{General information}

A total of 23 patients with advanced TNBC in the Guangxi Medical University Cancer Hospital were recruited between January 2017 and August 2019. Two cases were excluded due to giving up treatment after 1 cycle. Finally, 21 cases were included in the analysis (Figure 1) and their 


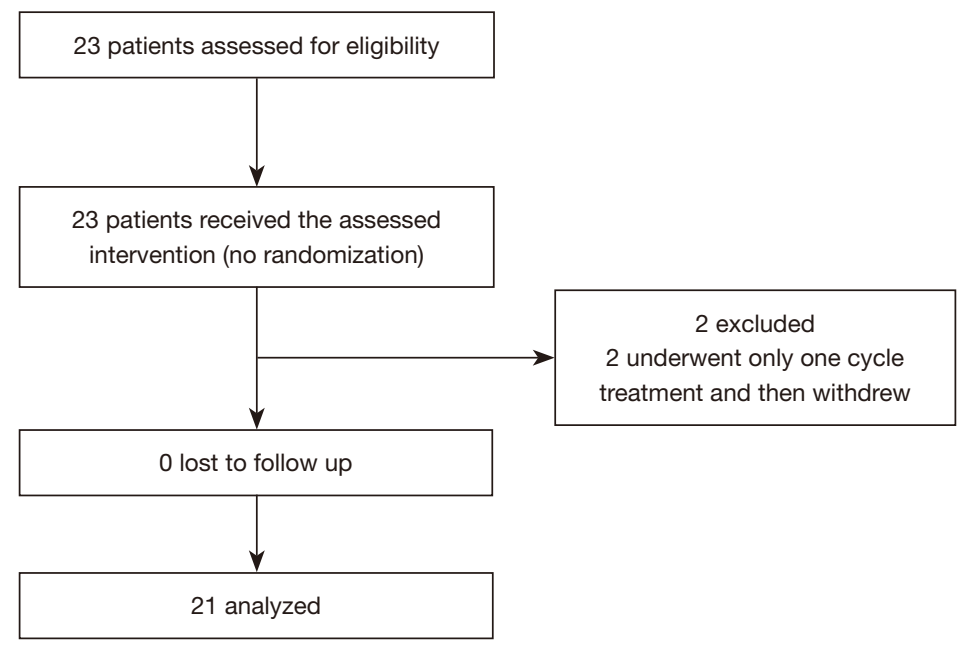

Figure 1 Flowchart of the participants through the trial (CONSORT diagram).

characteristics are listed in Table 1. The median age was 51 [interquartile range (IQR), 42-57] years. Among them, $11(52.4 \%)$ patients had $\geq 3$ metastatic organ sites, and visceral involvement was noted in 17 patients $(81.0 \%)$. Twelve (57.1\%) patients had no previous chemotherapies for metastatic disease. For the included TNBC patients $(n=17)$ initially diagnosed as early stage, all of them had received anthracycline or taxane treatment in the adjuvant stage. Among them, 4 patients (23.5\%) had disease progression within 6 months of the end of adjuvant chemotherapy (defined as resistance to anthracyclines or taxanes), and 7 patients $(41.2 \%)$ progressed after 1 year from the end of adjuvant chemotherapy (defined as sensitivity to anthracyclines or taxanes). For the included TNBC patients $(n=4)$ initially diagnosed as stage IV, 3 of them $(75.0 \%)$ had disease progression within 3 months of the first-line use of anthracyclines or taxanes (defined as resistance to anthracyclines or taxanes). The chemotherapy drugs combined with platinum included gemcitabine $(n=2)$, docetaxel $(n=4)$, and pemetrexed $(n=15)$.

\section{Clinical efficacy}

The median treatment cycle was 5 (range, 2-11 cycles). Treatment efficacy is summarized in Table 2 and Figure 2. The ORR and CBR for the included patients were $47.6 \%$ and $52.4 \%$, respectively. The ORRs for first-line and second-line or beyond were $50 \%$ and $44.4 \%$, respectively $(\mathrm{P}=0.801)$. For patients sensitive or resistant to anthracycline and taxane drugs, the ORRs were the same (42.9\%).
Although it was not statistically significant, a numerically higher CBR $(\mathrm{PR} / \mathrm{SD}>6 \mathrm{~m})(66.7 \%)$ was observed in patients who had not received any chemotherapy in the metastatic setting. In addition, a significantly higher CBR $(85.7 \%)$ was observed in patients who were sensitive to anthracycline and taxane drugs, compared to the $28.6 \%$ observed in those who were resistant to anthracycline and taxane drugs $(\mathrm{P}=0.031)$. The ORR and $\mathrm{CBR}$ were not different between patients who received pemetrexed combined with platinum or gemcitabine/taxanes combined with platinum (all $\mathrm{P}>0.05$ ). The last follow-up date was April 30, 2020. The median PFS (mPFS) was 8.8 months (95\% CI: 7.2-10.4 months) (Figure $3 A$ ) and the median OS was 13.3 months (95\% CI: 11.6-15.0 months) (Figure 3B). In addition, patients who were sensitive to anthracycline and taxane drugs showed a significantly longer mPFS compared to those who were resistant to anthracycline and taxane drugs (mPFS: 8.8 vs. 5.3 months, $\mathrm{P}=0.038$ ) (Figure $4 A$ ). For patients who received first-line therapy and secondline therapy or beyond, the mPFS was 8.8 and 5.3 months, respectively, with a significant difference $(\mathrm{P}=0.025)$ (Figure 4B). No statistically significant difference in the mPFS between pemetrexed combined with platinum and gemcitabine/taxanes combined with platinum was observed (Figure 4C).

\section{Toxic effects}

The most common drug-related AEs are shown in Table 3. The most common grade 3-4 hematologic toxicities 
Table 1 Patient characteristics $(\mathrm{n}=21)$

\begin{tabular}{|c|c|c|}
\hline Characteristics & Number & $\%$ \\
\hline Median [IQR] & \multicolumn{2}{|c|}{$51[42-57]$} \\
\hline \multicolumn{3}{|l|}{ ECOG status } \\
\hline $0-1$ & 16 & 76.2 \\
\hline \multicolumn{3}{|l|}{ Metastatic sites } \\
\hline Liver & 10 & 47.6 \\
\hline Lung & 11 & 52.4 \\
\hline Brain & 3 & 14.3 \\
\hline Chest wall & 4 & 19.0 \\
\hline Breast & 5 & 23.8 \\
\hline Bone & 8 & 38.1 \\
\hline Visceral & 17 & 81.0 \\
\hline Non-visceral & 4 & 19.0 \\
\hline \multicolumn{3}{|l|}{ Number of metastatic sites } \\
\hline 1 & 3 & 14.3 \\
\hline 2 & 7 & 33.3 \\
\hline \multicolumn{3}{|l|}{ Previous chemotherapy drugs } \\
\hline \multicolumn{3}{|c|}{ Adjuvant setting ( $\mathrm{n}$ of early-stage $B C=17$ ) } \\
\hline Anthracycline & 16 & 94.1 \\
\hline Taxane & 15 & 88.2 \\
\hline Anthracycline or taxane & 17 & 100.0 \\
\hline Both & 14 & 82.4 \\
\hline \multicolumn{3}{|c|}{ Metastatic setting ( $\mathrm{n}$ of patients treated $=9$ ) } \\
\hline Anthracycline & 4 & 44.4 \\
\hline Taxane & 7 & 77.8 \\
\hline Anthracycline or taxane & 7 & 77.8 \\
\hline Both & 4 & 44.4 \\
\hline
\end{tabular}

Table 1 (continued) 
Table 1 (continued)

\begin{tabular}{|c|c|c|}
\hline Characteristics & Number & $\%$ \\
\hline DFS $<6 \mathrm{~m}$ & 4 & 23.5 \\
\hline $6 \mathrm{~m} \leq \mathrm{DFS} \leq 12 \mathrm{~m}$ & 6 & 35.3 \\
\hline DFS $>12 \mathrm{~m}$ & 7 & 41.2 \\
\hline Primary resistance to anthracycline and taxane & 3 & 75.0 \\
\hline Unknown & 1 & 25.0 \\
\hline \multicolumn{3}{|l|}{ Combined chemotherapy } \\
\hline Gemcitabine & 2 & 9.5 \\
\hline
\end{tabular}

IQR, interquartile range; ECOG, Eastern Cooperative Oncology Group; BC, breast cancer; DFS, disease-free survival.

Table 2 Summary of treatment efficacy

\begin{tabular}{|c|c|c|c|c|c|c|c|c|c|c|}
\hline Variables & $N$ & CR & PR & SD & PD & ORR, \% & $P$ value & \multicolumn{3}{|c|}{$\mathrm{PR} / \mathrm{SD}>6 \mathrm{~m}$} \\
\hline Whole group & 21 & 0 & 10 & 8 & 3 & 47.6 & & 11 & 52.4 & \\
\hline \multicolumn{11}{|l|}{ Lines of treatment } \\
\hline First-line & 12 & 0 & 6 & 4 & 2 & 50.0 & 0.801 & 8 & 66.7 & 0.130 \\
\hline \multicolumn{11}{|c|}{$\begin{array}{l}\text { Sensitivity to anthracyclines } \\
\text { or taxane }\end{array}$} \\
\hline Yes & 7 & 0 & 3 & 4 & 0 & 42.9 & 1.00 & 6 & 85.7 & 0.031 \\
\hline No & 7 & 0 & 3 & 2 & 2 & 42.9 & & 2 & 28.6 & \\
\hline
\end{tabular}

$\mathrm{CR}$, complete response; PR, partial response; SD, stable disease; PD, progressive disease; ORR, objective response rate; CBR, clinical benefit rate.

were neutropenia $(14.3 \%)$ and anemia $(14.3 \%)$. One patient $(4.8 \%)$ experienced febrile neutropenia. No grade 3-4 non-hematologic toxicities were observed. Rhendostatin-related AEs included hypertension (14.3\%) and proteinuria (19.0\%). However, only 1 patient who had essential hypertension experienced grade 3 hypertension. The others all experienced grade 1-2 hypertension or proteinuria. No epistaxis was observed. Dose adjustment due to AEs was performed for 2 patients $(9.5 \%)$. No treatmentrelated deaths were occurred. Overall, the toxicities of the combination therapy were acceptable and manageable.

\section{Discussion}

Although great progress has been made in the development of new therapeutic agents, the OS of TNBC remains poor. 


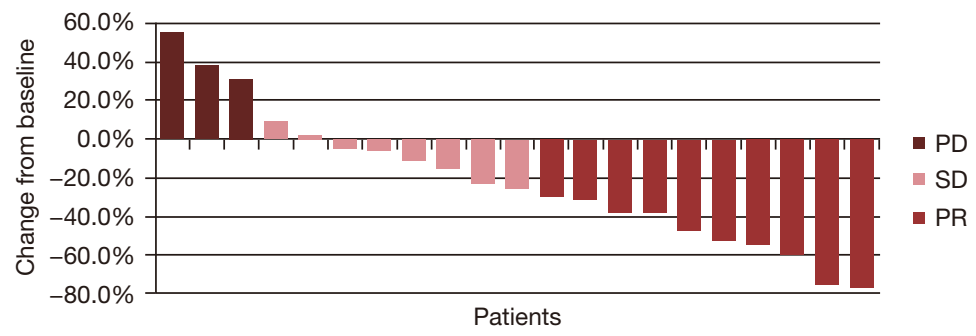

Figure 2 Best overall response. PD, progressive disease; SD, stable disease; PR, partial response.

A

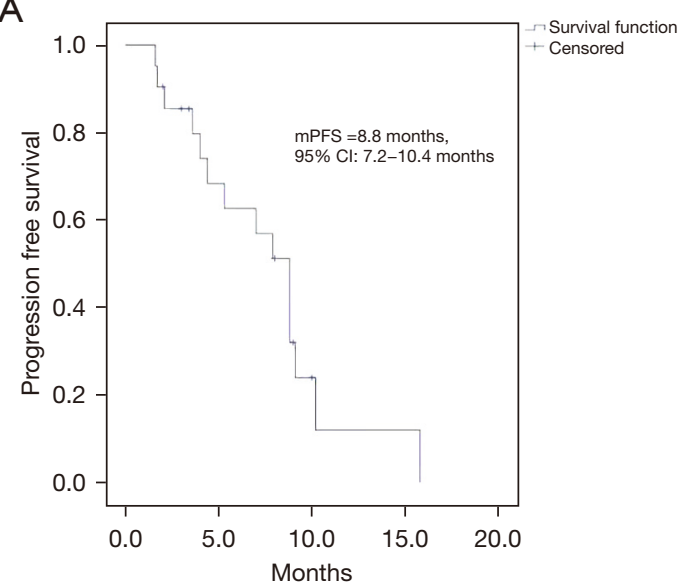

B

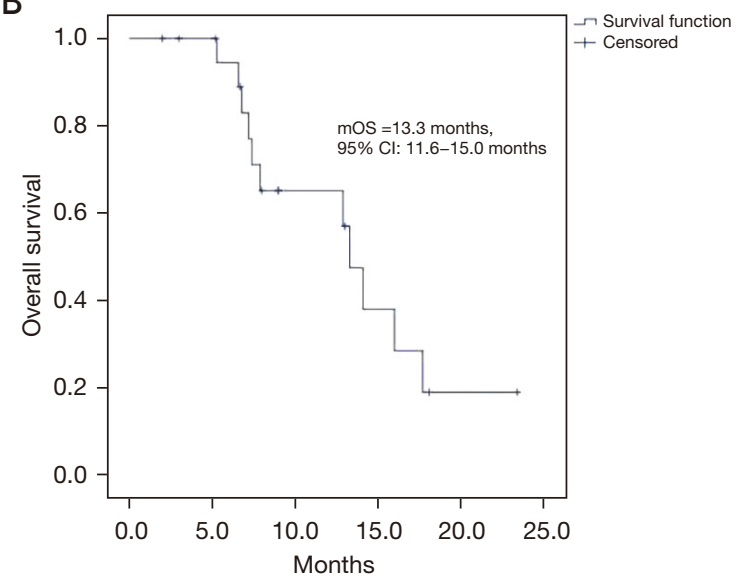

Figure 3 Kaplan-Meier estimates. (A) The overall PFS; (B) the OS. PFS, progression-free survival; OS, overall survival; mPFS, median PFS.

It has been well acknowledged that neoangiogenesis plays an important role in cancer progression, and TNBC is characterized by high vascularity $(5,6)$, indicating that antiangiogenesis may be a promising therapeutic strategy for treating advanced TNBC. This study investigated the efficacy of Rh-endostatin, a vascular epithelial inhibitor, combined with platinum-based chemotherapy in the treatment of patients with advanced TNBC, which might help optimize the current management.

Due to the lack of a clear and effective therapeutic target, chemotherapy is currently still the mainstay of systemic treatment for TNBC. Anthracyclines and taxanes are the preferred drugs for $\mathrm{BC}$, but they are often used in neoadjuvant or adjuvant chemotherapy. In this study, all patients received anthracyclines or taxanes at the adjuvant stage. It was reported that TNBC commonly harbors BRCA mutations, and over $75 \%$ of $B R C A 1 / 2$-mutated BCs show the TNBC phenotype (3), which makes TNBC susceptible to DNA-damaging compounds such as platinum drugs. The commonly used platinum agents for $\mathrm{BC}$ are carboplatin and cisplatin $(15,16)$. Therefore, carboplatin/carboplatin-based chemotherapy is an effective rescue chemotherapy regimen for advanced TNBC. To date, many studies have reported the clinical application of platinum-based chemotherapy in TNBC, but the PFS ranged between 5.3-7.8 months and the ORR ranged between $33.3-37.5 \%$ in the first-line metastatic setting $(15,17-20)$. In view of this, more studies need to be conducted to optimize the treatment efficacy. $\mathrm{Rh}$-endostatin is a new recombinant human endostatin developed in China, which has been shown to inhibit tumor endothelial cell proliferation, angiogenesis, and tumor growth (21). Clinical studies reported that Rh-endostatin showed both favorable safety and efficacy profile in treating several kinds of human cancers (8). Mechanism studies showed that Rh-endostatin induced vascular normalization to improve chemotherapy efficacy through suppression of Src signaling pathway (22). And vascular normalizing does of antiangiogenic treatment could directly alleviate hypoxia and make the tumor immune environment changed from an immunosuppressive state to an immune- 
A
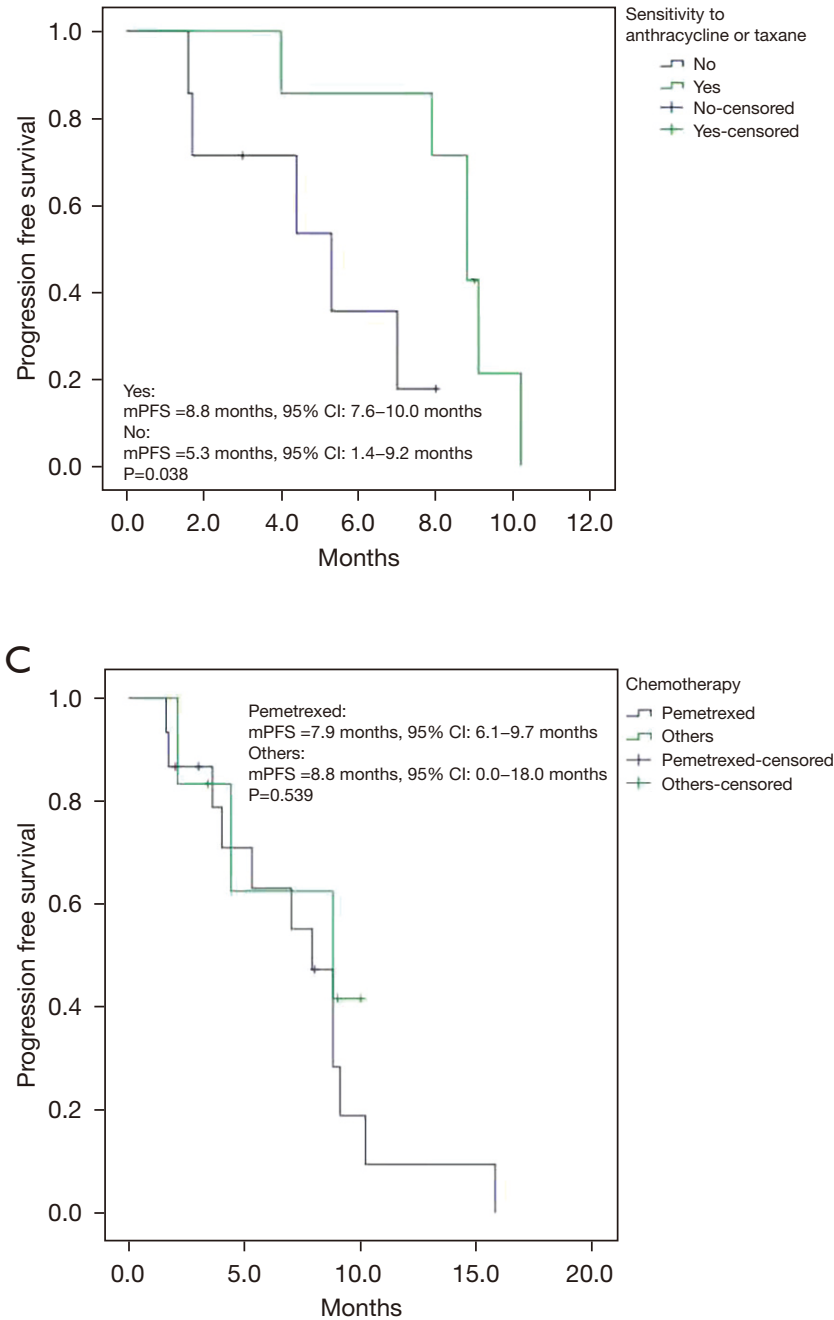

B

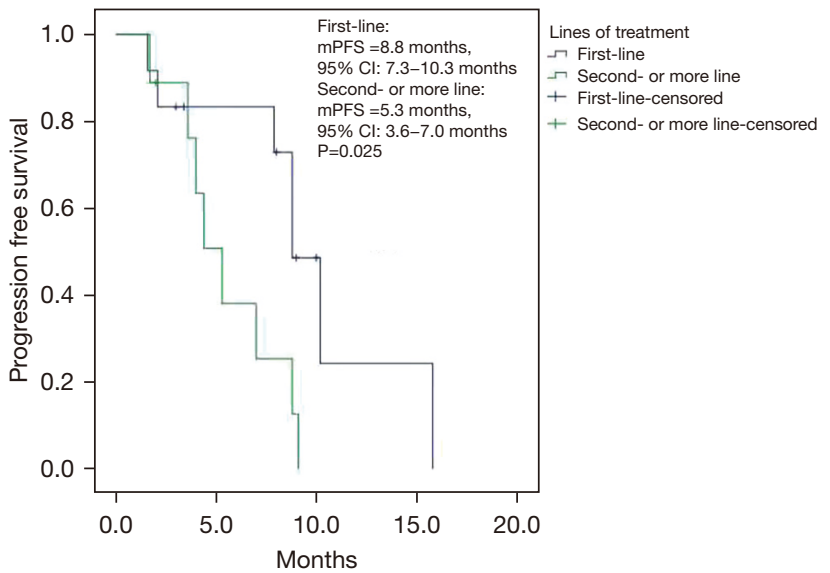

Figure 4 The stratified analysis. (A) Sensitivity to anthracyclines or taxanes; (B) the number of treatment lines; (C) the combined treatment regimens. PFS, progression-free survival; mPFS, median PFS.

supportive one (23). Rh-endostatin in combination with platinum-based chemotherapy showed synergic action and a favorable toxicity profile in advanced NSCLC patients (24). The combination of Rh-endostatin and cisplatin/etoposide in patients with extensive-stage small-cell lung cancer (EDSCLC) resulted in slightly improved PFS and OS relative to historical controls who received this chemotherapy regimen alone (25). Results by Jin et al. revealed that Rh-endostatin combined with gemcitabine and cisplatin chemotherapy (E $+\mathrm{GP}$ ) was an effective, well-tolerated regimen for metastatic nasopharyngeal carcinoma (M-NPC) (26). Furthermore, Rh-endostatin combined with chemotherapy had significant activity to increase the PFS and improve CBR in patients with advanced sarcomas with tolerable side effects (27). However, few studies were related with the treatment of advanced TNBC using Rh-endostatin. Considering the potential therapeutic benefits of combination of Rh-endostatin plus platinum-based chemotherapy, we conducted this study. This study explored the efficacy of Rhendostatin combined with platinum-based chemotherapy in advanced TNBC patients, revealing that Rh-endostatin combined with platinum-based chemotherapy had a PFS of 8.8 months and an ORR of $47.6 \%$, which were better than the previously reported efficacy rates of platinumbased chemotherapy alone (15,17-20). The results were basically consistent with a previously reported clinical 
Table 3 AEs $(\mathrm{n}=21)$

\begin{tabular}{lccc}
\hline AEs & All grades, $\mathrm{n}(\%)$ & Grade 1 or 2, $\mathrm{n}(\%)$ & Grade 3 or 4, $\mathrm{n}(\%)$ \\
\hline Hematologic & & & $2(9.5)$ \\
Leukopenia & $14(66.7)$ & $12(57.1)$ & $3(14.3)$ \\
Neutropenia & $13(61.9)$ & $10(47.6)$ & $1(4.8)$ \\
Febrile neutropenia & $1(4.8)$ & $\mathrm{NA}$ & $3(14.3)$ \\
Anemia & $12(57.1)$ & $9(42.9)$ & $2(9.5)$ \\
Thrombocytopenia & $4(19.0)$ & $2(9.5)$ & $0(0.0)$ \\
Non-hematologic & & & $0(0.0)$ \\
Peripheral neurotoxicity & $5(23.8)$ & $5(23.8)$ & $0(0.0)$ \\
Fatigue & $3(14.3)$ & $3(14.3)$ & $0(0.0)$ \\
Nausea & $6(28.6)$ & $6(28.6)$ & $0(0.0)$ \\
Vomiting & $2(9.5)$ & $2(9.5)$ & $0(0.0)$ \\
Diarrhea & $2(9.5)$ & $2(9.5)$ & $1(4.8)^{\star}$ \\
Liver dysfunction & $9(42.9)$ & $9(42.9)$ & $0(0.0)$ \\
Hypertension & $3(14.3)$ & $2(9.5)$ & $4(19.0)$ \\
Proteinuria & $4(19.0)$ & & \\
\hline
\end{tabular}

*, this patient has hypertension. AEs, adverse events.

study of the anti-angiogenic drug bevacizumab combined with chemotherapy in advanced TNBC $(28,29)$. Further stratified analyses showed that Rh-endostatin combined with platinum-based first-line treatment was superior to post-line treatment, and the benefits were greater in anthracycline and taxane sensitive groups, which was consistent with previous research reports (30). Furthermore, this study analyzed the application of pemetrexed combined chemotherapy in advanced TNBC, indicating that when compared with conventional chemotherapy drugs for BC (such as gemcitabine and docetaxel), there were no differences in PFS or ORR between the two groups. These results indicated that pemetrexed might also be an alternative drug for the treatment of TNBC. However, the sample size of this study was small, and large, prospective, and controlled studies should be designed for further analysis.

In general, TNBC is a highly heterogeneous disease with diverse clinical courses, and pathological, molecular and genetic features. According to Jiang et al. (31), primary Chinese TNBC was characterized and classified it into four subtypes with putative therapeutic targets: (I) luminal androgen receptor (LAR); (II) immunomodulatory (IM); (III) basal-like immune-suppressed (BLIS) and
(IV) mesenchymal-like (MES). Endocrine therapy and targeting ERBB2 were recommended for LAR TNBC subtype, with immune checkpoint inhibitors (ICI) recommended for IM TNBC subtype. Besides, platinum drugs were recommended for BLIS TNBC subtype, with STAT3 inhibitor recommended for MES TNBC subtype. Therefore, it was necessary to identify profiles and biomarkers to further investigate the treatment efficacy of Rh-endostatin. Furthermore, experimental data demonstrated that the antitumor activity of Rh-endostatin was time and dose dependent. It was observed that a micropump could maintain an effective concentration of Rh-endostatin for a long time, which is a more effective delivery mode in the treatment of tumors (32). A recent study (33) reported that Rh-endostatin continuous infusion combined with chemotherapy was effective in the treatment of advanced NSCLC, with tolerable AEs. In this study, patients were treated with a 24-hour continuous infusion of Rh-endostatin through a micro-infusion pump for 7 days, which, combined with platinum-based chemotherapy, improved PFS, yet did not increase the incidences of AEs. This treatment model minimized the hospitalization, so as to improve patient compliance. 


\section{Limitations}

The limitations in this study should not be neglected. First, the sample size of this study was small. Due to limited time and fund, it was difficult to accumulate a lot of clinical cases that meet the inclusion criteria in a relatively short period of time. Besides, it was of great urgency to explore and unveil alternative treatment option for TNBC patients. Hence, only a limited number of clinical cases were involved in this preliminary attempt. Second, limited-stage patients with heterogeneity were enrolled in this study, which might lead to some uncertainty of the therapeutic effects. Besides, this study was a single-arm, single-center, nonblind study with no control group, which could inevitably lead to some biases. Therefore, more clinical cases will be continuously accumulated to conduct larger sample size, multi-center, randomized and controlled clinical trials to provide definitive validation for the use of Rh-endostatin as a follow-up strategy against advanced TNBC, as well as to confirm its long-term efficacy and toxicity in the future.

\section{Conclusions}

In conclusion, this study provided evidences that $\mathrm{Rh}$ endostatin might enhance the antitumor effects of platinum-based chemotherapy for advanced TNBC patients with well-tolerated toxicities, which demonstrated that Rhendostatin combined with platinum-based chemotherapy might be a new feasible treatment for advanced TNBC. In the follow-up, we will further explore the maintenance regimen, duration, and patients who will benefit most.

\section{Acknowledgments}

Funding: None.

\section{Footnote}

Reporting Checklist: The authors have completed the TREND reporting checklist. Available at https://dx.doi. org/10.21037/apm-21-2624

Data Sharing Statement: Available at https://dx.doi. org/10.21037/apm-21-2624

Conflicts of Interest: All authors have completed the ICMJE uniform disclosure form (available at https://dx.doi. org/10.21037/apm-21-2624). The authors have no conflicts of interest to declare.

Ethical Statement: The authors are accountable for all aspects of the work in ensuring that questions related to the accuracy or integrity of any part of the work are appropriately investigated and resolved. All of the enrolled patients or their family members signed the informed consent and could actively cooperate with the treatment. All procedures performed in this study involving human participants were in accordance with the Declaration of Helsinki (as revised in 2013). The study was approved by the Medical Ethics Committee of Guangxi Medical University Cancer Hospital (No. LW2021099).

Open Access Statement: This is an Open Access article distributed in accordance with the Creative Commons Attribution-NonCommercial-NoDerivs 4.0 International License (CC BY-NC-ND 4.0), which permits the noncommercial replication and distribution of the article with the strict proviso that no changes or edits are made and the original work is properly cited (including links to both the formal publication through the relevant DOI and the license). See: https://creativecommons.org/licenses/by-nc-nd/4.0/.

\section{References}

1. Zhao S, Zuo WJ, Shao ZM, et al. Molecular subtypes and precision treatment of tri-ple-negative breast cancer. Ann Transl Med 2020;8:499.

2. Caramelo O, Silva C, Caramelo F, et al. The effect of neoadjuvant platinum-based chemotherapy in BRCA mutated triple negative breast cancers -systematic review and meta-analysis. Hered Cancer Clin Pract 2019;17:11.

3. Guney Eskiler G, Cecener G, Egeli U, et al. Triple negative breast cancer: new therapeutic approaches and BRCA status. APMIS 2018;126:371-9.

4. Chen J, Liu BX, Shen Q, et al. Limonin inhibits angiogenesis and metastasis of human breast cancer cells by suppressing the VEGFR2/IGFR1-mediated STAT3 signaling path-way. Transl Cancer Res 2020;9:6820-32.

5. Zhao Z, Li Y, Shukla R, et al. Development of a Biocompatible Copolymer Nanocomplex to Deliver VEGF siRNA for Triple Negative Breast Cancer. Theranostics 2019;9:4508-24.

6. Ribatti D, Nico B, Ruggieri S, et al. Angiogenesis and Antiangiogenesis in Triple-Negative Breast cancer. Transl Oncol 2016;9:453-7.

7. $\mathrm{Xu}$ X, Mao W, Chen Q, et al. Endostar, a modified 
recombinant human endostatin, suppresses angiogenesis through inhibition of $\mathrm{Wnt} / \beta$-catenin signaling pathway. PLoS One 2014;9:e107463.

8. Li K, Shi M, Qin S. Current Status and Study Progress of Recombinant Human Endostatin in Cancer Treatment. Oncol Ther 2018;6:21-43.

9. Huang W, Liu J, Wu F, et al. The efficacy and safety of endostar combined with taxane-based regimens for HER2-negative metastatic breast cancer patients. Oncotarget 2016;7:31501-7.

10. Chen J, Yao Q, Li D, et al. Neoadjuvant rh-endostatin, docetaxel and epirubicin for breast cancer: efficacy and safety in a prospective, randomized, phase II study. BMC Cancer 2013;13:248.

11. Zhang X, Zhang Z, Cao M, et al. A Randomized Parallel Controlled Phase II Trial of Recombinant Human Endostatin Added to Neoadjuvant Chemotherapy for Stage III Breast Cancer. Clin Breast Cancer 2020;20:291-9.e3.

12. Zhu F, Tang XF, Sun JB. Observation and nursing of different administration methods of Endostar combined with chemotherapy for advanced non-small cell lung cancer. Fujian Medical Journal 2019;41:156-8.

13. Schwartz LH, Litière $S$, de Vries E, et al. RECIST 1.1-Update and clarification: From the RECIST committee. Eur J Cancer 2016;62:132-7.

14. Common Terminology Criteria for Adverse Events (CTCAE) Version 4.0. Available online: https://evs. nci.nih.gov/ftp1/CTCAE/CTCAE_4.03/Archive/ CTCAE_4.0_2009-05-29_QuickReference_8.5x11.pdf (Accessed 25-11-2019).

15. Staudacher L, Cottu PH, Diéras V, et al. Platinum-based chemotherapy in metastatic triple-negative breast cancer: the Institut Curie experience. Ann Oncol 2011;22:848-56.

16. Chen Y, Zhang J, Hu XC, et al. Maintenance chemotherapy is effective in patients with metastatic triple negative breast cancer after first-line platinum-based chemotherapy. Ann Palliat Med 2020;9:3018-27.

17. Zhang J, Fan M, Xie J, et al. Chemotherapy of metastatic triple negative breast cancer: Experience of using platinumbased chemotherapy. Oncotarget 2015;6:43135-43.

18. Hu XC, Zhang J, Xu BH, et al. Cisplatin plus gemcitabine versus paclitaxel plus gemcitabine as first-line therapy for metastatic triple-negative breast cancer (CBCSG006): a randomised, open-label, multicentre, phase 3 trial. Lancet Oncol 2015;16:436-46.

19. Jin J, Zhang W, Ji W, et al. Predictive biomarkers for triple negative breast cancer treated with platinum-based chemotherapy. Cancer Biol Ther 2017;18:369-78.
20. Koshy N, Quispe D, Shi R, et al. Cisplatin-gemcitabine therapy in metastatic breast cancer: Improved outcome in triple negative breast cancer patients compared to nontriple negative patients. Breast 2010;19:246-8.

21. Xu H, Lv D, Meng Y, et al. Endostar improved efficacy of concurrent chemoradiothera-py with vinorelbine plus carboplatin in locally advanced lung squamous cell carcinoma patients with high serum $\mathrm{Lp}$ (a) concentration. Ann Palliat Med 2020;9:298-307.

22. Yu M, Han Y, Zhuo H, et al. Endostar, a Modified Endostatin Induces Vascular Normalization to Improve Chemotherapy Efficacy Through Suppression of Src Signaling Pathway. Cancer Biother Radiopharm 2018;33:131-8.

23. Huang Y, Yuan J, Righi E, et al. Vascular normalizing doses of antiangiogenic treatment reprogram the immunosuppressive tumor microenvironment and enhance immunotherapy. Proc Natl Acad Sci U S A 2012;109:17561-6.

24. Sun Y, Wang JW, Liu YY, et al. Long-term results of a randomized, double-blind, and placebo-controlled phase III trial: Endostar (rh-endostatin) versus placebo in combination with vinorelbine and cisplatin in advanced non-small cell lung cancer. Thorac Cancer 2013;4:440-8.

25. Zhou ZT, Zhou FX, Wei Q, et al. Phase II study of cisplatin/etoposide and endostar for extensive-stage small-cell lung cancer. Cancer Chemother Pharmacol 2011;68:1027-32.

26. Jin T, Jiang F, Jin QF, et al. Endostar Combined with Gemcitabine and Cisplatin Chemotherapy for Patients with Metastatic Nasopharyngeal Carcinoma: an Update. Transl Oncol 2018;11:286-91.

27. Xing $\mathrm{P}$, Zhang J, Yan Z, et al. Recombined humanized endostatin (Endostar) combined with chemotherapy for advanced bone and soft tissue sarcomas in stage IV. Oncotarget 2017;8:36716-27.

28. Hamilton E, Kimmick G, Hopkins J, et al. Nab-paclitaxel/ bevacizumab/carboplatin chemotherapy in first-line triple negative metastatic breast cancer. Clin Breast Cancer 2013;13:416-20.

29. Mery B, Rowinski E, Vallard A, et al. Advocacy for a New Oncology Research Paradigm: The Model of Bevacizumab in Triple-Negative Breast Cancer in a French Cohort Study. Oncology 2019;97:1-6.

30. Seah DS, Luis IV, Macrae E, et al. Use and duration of chemotherapy in patients with metastatic breast cancer according to tumor subtype and line of therapy. J Natl Compr Canc Netw 2014;12:71-80. 
31. Jiang YZ, Ma D, Suo C, et al. Genomic and Transcriptomic Landscape of Triple-Negative Breast Cancers: Subtypes and Treatment Strategies. Cancer Cell 2019;35:428-40.e5.

32. Li W, Zhao X, Du B, et al. Gold Nanoparticle-Mediated Targeted Delivery of Recombinant Human Endostatin Normalizes Tumour Vasculature and Improves Cancer Therapy. Sci Rep 2016;6:30619.

Cite this article as: Tan A, Wang $\mathrm{H}$, Nong L, Jia Y, Liu Y, Zhong W, Qin F, Wang H, Tang J, Zhou W, Lu Y, Xie W. Efficacy and safety of continuous infusion of Rh-endostatin combined with platinum-based chemotherapy for advanced triple-negative breast cancer. Ann Palliat Med 2021;10(12):1210112112. doi: 10.21037/apm-21-2624
33. Cheng Y, Nie L, Liu Y, et al. Comparison of Endostar continuous versus intermittent intravenous infusion in combination with first-line chemotherapy in patients with advanced non-small cell lung cancer. Thorac Cancer 2019;10:1576-80.

(English Language Editor: C. Betlazar-Maseh) 